\title{
The participatory approach in Morocco against the 2011 constitution: Lure or reality
}

\author{
Si Ahmed Attaf
}

Department of Geography, Ibn Tofail University, Faculty of Letters and Human Sciences, Morocco

Received: 1 Oct 2020; Received in revised form: 8 Nov 2020; Accepted: 10 Nov 2020; Available online: 22 Nov 2020

(C)2020 The Author(s). Published by Infogain Publication. This is an open access article under the CC BY license

(https://creativecommons.org/licenses/by/4.0/).

\begin{abstract}
This study aims to focus on a fairly new concept in participatory democracy, called citizen participation or participatory approach supposed to impact on the decision-making process of public authorities in terms of the needs or needs of the population, by putting the citizen in the spotlight. At the center of this process is at least what our constitution lets deduce, the article therefore promises to give answers to the following questions: First, what is the difference between representative democracy and participatory democracy? Deuzio, what are the procedures and legislative mechanisms available to citizens to exercise this right? Third, what are the limits that hamper the efficiency of the participatory approach? This analytical study a fortiori, and the experiences have revealed the limits of this approach in the materialization of the real needs of citizens who are often poorly consulted. the citizen law and the public authorities share the responsibility for these obstacles.
\end{abstract}

Keywords-Participatory approach, citizen, constitution, constraints, representative democracy.

\section{L'approche participative au Maroc à l'aunede la constitution de 2011: Leurre ou réalité}

\begin{abstract}
Cette étude a pour objectifs de mettre l'accent sur un concept assez nouveau dans la démocratie participative, dit participation citoyenne ou approche participative censée impacter sur le processus décisionnel des autorités publiques en matière de nécessités ou besoins de la population, en mettant le citoyen au centre de ce processus c'est du moins ce que laisse déduire notre constitution, l'article se promet donc, de donner des réponses aux interrogations qui suivent: Primo, quelle estla différence entre la démocratie représentative et la démocratie participative? Deuzio, quels sontles procédés et mécanismes législatifs mis à la portée du citoyen pour exercer ce droit? Tertio, quelles sont les limites qui cabrent l'efficience de l'approche participative? Cette étude analytique à fortiori,et les expériences ont révélé les limites de cette approche dans la matérialisation des besoins réels des citoyennes et citoyens souvent mal consultés. Le citoyen, la loi et les autorités publiques se partagent la responsabilité desdits entraves.
\end{abstract}

Les mots-clés - Approche participative, citoyen, constitution, contraintes, démocratie représentative.

\section{INTRODUCTION}

D'aucun ne peut nier le rôle prédominant de l'Etat dans la gestion de la chose publique, ce dernier a toujours été l'acteur principal dans toute politique publique, se portant garant de la bonne marche des différents services publics de l'Etat en guise d'une satisfaction de la demande, d'abord, interne qui répond aux aspirations des citoyens et qui maintient la stabilité des différentes institutions étatiques à 
même de contribuer au développement des conditions des administrations et des administrés, puis international epermettant à l'Etat se démarquer sur la scène mondiale, et de supporter le poids d'une concurrence de plus en plus accrue et pesante.

La gestion publique,que ce soit au niveau national service publics nationaux-, ou de proximité - service publics locaux-, semble être le pilier de la réussite ou non des politiques publiques dans le cadre d'une vision globale basée sur d'efficientes stratégies où la bonne gouvernance sert de locomotive,laquelle requiert, désormais,plus d'implication des citoyens à la prise de décision, partant d'une approche participative, fondement universel des démocraties modernes, mettant le citoyen, et la société civile au centre de l'exercice démocratique, sans se départir de l'approche genre ;car la construction, la concrétisation et l'incidence des politiques publiques sont censées impacter en final sur le citoyen lui-même, équitablementet quelques soit son sexe, son statut ou ses besoins. C'est sans doute pour cette raison que la constitution marocaine de 2011, en a fait un cheval de bataille.Dès lors, de multiples interrogations s'imposent :La consécration constitutionnelle de l'approche participative suffirait-elle pour impliquer réellement le citoyenhabitué jusque-làà être relégué au second degré ?Les lois organiques censées matérialiser l'esprit de la constitution entermes de participation locale,ont-ilsréussi à rendre tangible laditeapproche ? D'autre part,quelles sont les limites qui se laisseront déduire du même texte et pouvant ultérieurement surgir pour cabrer la capacité participative et par conséquent, décisionnelle ducitoyen? Les limites se percevraient-elles uniquement au niveau des textes ou le citoyen lui-même endosserait-il une part de responsabilité ?

Dans une perspective d'apporter des réponses à cette problématique,nous tenterons,via une approche analytique des textes, associant par moment la pratique,dedissiper, dans un premier lieu, la confusion entre les concepts :représentation et participation, dans un second lieu, nous mettrons la lumière sur le cadre juridique consacrant l'approche participative au Maroc, pour aboutir, dans un troisième, à en préciser les contraintes inhérentes à sa mise en œuvre.

\section{LA DEMOCRATIE REPRESENTATIVE ET LA DEMOCRATIE PARTICIPATIVE}

Il s'agit là de deux notions voire deux concepts tout à fait différents et qui, pourtant,s'emploient confusément,dans la mesure où une grande partie de la population a tendance à les associer fréquemmentaux élections.

\subsection{La démocratie représentative}

«Le citoyen », statut à portée large ou «l'électeur»,partant d'une acception plus spécifique et plus institutionnelle, dans une démocratie représentative, choisit celui qui va le représenter, en l'occurrence,le représentant qui se substitue à lui et en devient tuteur, «le corps électoral désigne, les représentants décident $»^{1}$

Le «représentant», d'une optique purement politique,a deux facettes : il est celui qui incarne le «représenté »et celui qui agit en son nom. Or, dans les deux situations, s'il rend l'autre présent, c'est à condition de se substituer à lui, dès lors, le représenté se trouve dans une position passiveescamotépar celui qui le représente. Ce constat est tellement véridique que dans un État représentatif, le peuple ne peut parler et ne peut agir que parses représentants. ${ }^{2}$ Le représentant est donc libre de ses décisions. Les électeurs ne lui tracent pas de programme; ils s'en remettent à lui pour discerner les meilleurs décisions, il n'a d'autres engagement que d'agir en conscience, dans le cas de changement dans ses opinons il est irrévocable ${ }^{3}$, il jouit donc d'une investiture quasi absolue pendant toute la durée du mandat,la démocratie représentative nous renvoie, en ce sens, automatiquement à la conception électorale, nous retenons donc la définition adoptée parle ministère chargé des relations avec le gouvernement et la société civile marocainlors d'un colloque datant du 04 Avril 2016: « la démocratie représentative, est l'une des formes de la démocratie dans laquelle les citoyens expriment leur volonté par l'intermédiaire de représentants élus à qui ils délèguent leurs pouvoirs ».

Qu'en est-il alors deson corollaire, la démocratie participative !?

\subsection{La démocratie participative}

Au cours des dernières décennies, deux phénomènes importants ont marqué le paysage politique, l'avènement de nouveaux acteurs sociaux qui revendiquent une parcelle dans la scène politique, le sentiment d'insatisfaction de la population à l'égard des institutions et des politiques publiques. En réponse, les gouvernements se sont engagés, à revoir leurs mécanismes de prise de décisions politiques en prétendant à instituer de nouveaux espaces publics et de nouveaux mécanismes destinés à favoriser la concertation publique. C'est alors, que la notion de la participation citoyenne qui renvoie directement àl'approche participative, fait figure dans les débats politiques et commence à prendre place, petit à petit. Elle désigneun processus d'engagement obligatoire ou volontaire des personnes ordinaires, agissant seules ou au sein d'une organisation, en vue d'influer sur une décision portant sur 
des choix significatifsqui toucheront leur communauté. Cette participation peut avoir lieu ou non dans un cadre institutionnalisé et être organiséesous l'initiative des membres de la société civile (recours collectif, manifestation, comités de citoyens) ou des décideurs (référendum,commissionparlementaire, médiation)4. Par extension, « la démocratie participative évoque l'idée d'une implication et d'une participation direct des citoyens dans le débat public et dans la prise de décisions politiques $» 5$.

Ainsi l'on déduit que le citoyen a le pouvoir de faire entendre sa voix et participer à la prise de décision sans condition aucune, pour confirmer ou infirmer ce constat, une lecture minutieuse des textes de loi s'avère des plus nécessaires, notamment la loi suprême et les autres textes qui en découlent.

\section{LE CADRE JURIDIQUE DE L'APPROCHE PARTICIPATIVE AU MAROC}

A l'ère actuelle, le Maroc se veut, plus que jamais, un Etat émergent capable de remplir les critères mondiaux requis pour se démarquer sur la scène internationale tout en les conciliant avec les exigences économiques, politiques et sociales propices à la demande interne et garante d'une réelle mutation démocratique. A cet effet une nouvelle constitution depuis 2011 a été démocratiquement adoptée, apportant de nouvelles garanties constitutionnelles, désireuses de créer un climat favorable à l'exercice démocratique, prenant en considération le projet pilote et ambitieux sur la régionalisation avancée réalisé avec une vision globale sur le nouveau statut de la région et ses nouvelles vocations, coiffée en parallèle par trois lois organiques organisant les trois échelons des collectivités territoriales respectivement: les régions, les provinces et les communes. Ce qui fait de la constitution et les lois organiques conséquentes notre cadre de référence en matière d'approche participative.

\subsection{La constitution de 2011}

\subsubsection{Contexte}

Il va sans dire que les demandes et les pressions sociales croissantes en 2010, mues par la mouvance contestataires dans la région arabe, en l'occurrence, l'éclosion depuis décembre 2010 du printemps arabe, n'ont pas été sans incidences sur la levée d'une vague de manifestations revendicatricesau Maroc, en parallèle, les hautes autorités du pays ont flairé le danger qui menaçait la stabilité nationale, ipso facto,on a diligenté une révision de la constitution qu'on a soumis au plébiscite populaire le 29 juillet 2011, nourriedu désir de donner raison aux mouvements revendicateurs qui prétendaient, entre autres, à la moralisation de la gestion publique, l'amélioration de ISSN: 2456-7620 l'offre en matière de prestations administratives, de nouvelles marges des libertés d'expression...etc.

Le pouvoir en place a œuvré pour que la nouvelle norme suprême puisse voir le jour, avec de nouvelles garanties d'une bonne gouvernance, un souci d'efficacité et d'efficience, et principalement une implication des citoyennes et citoyensmécontents, de plus en plus, dans le processus décisionnel de la gestion de la chose publiquepar la consécration des mécanismes propices à la participation citoyenne.

\subsubsection{La consécration constitutionnelle de l'approche participative}

Une lecture consciente de la constitution marocaine de 2011, permet de relever un nombre considérable d'articles afférents directement ou indirectement à l'approche participative,incluant l'approche genre, nous nous en tiendrons à une citation succinctedes différentes matièresy faisant allusion.D'abord, Le préambule qui stipule clairement dans le premier paragraphe que «le royaume poursuit résolument le renforcement des institutions d'un Etat moderne, ayant pour fondement les principes de participation, de pluralismes de bonne gouvernance», ensuite le premier article ajoute que «le régime constitutionnel du Royaume est fondé sur la séparation, l'équilibre et la collaboration des pouvoirs, ainsi que sur la démocratie citoyenne et participative .. », de même, les articles 12,13, 14, 15, évoquent respectivement «la contribution des organisations de la société civile dans l'élaboration, la mise en ouvre et l'évaluation des décisions et des projet des institutions élus et de pouvoirs publics. », " la création des instances de concertation, envue d'associer lesdifférents acteurs sociaux à l'élaboration, la mise en œuvre et l'évaluation des politiques publiques. », « ..droit de présenter des motions en matière législative. ${ }^{6}$, «les citoyennes et les citoyens disposent du droit de présenter des pétitions " ${ }^{7}, 1$ 'article 33, par conséquent, incitent les pouvoirs publics à «prendre toutes les mesures pour: - étendre et généraliser la participation de la jeunesse au développement social, économique, culturel et politique du pays. -aider les jeunes à s'insérer dans la vie active et associative.- faciliter l'accès des jeunes à la culture, à la science, à la technologie, à l'art, au sport et aux loisirs, tout en fixant les conditions propices au plein déploiement de leur potentiel créatif et innovant dans tous ces domaines.. - il est créeà cet effet un conseil consultatif de la jeunesse et l'action associative.. », plus loin, l'article 139 prévoit «lesmécanismes participatifs de dialogue et de concertation.. » que doivent favoriser les collectivités territoriales pour l'implication « des citoyennes et citoyens et des associations dans l'élaboration et le 
suivides programmes de développement ..» et en fin l'article 170 incitant à la création du «Conseil consultatif de la jeunesse et de l'action associative, une instance consultative dans des domaines de la protection de la jeunesse et de la promotion de la vie associative. ».

Utile,donc, est de rappeler au terme de la citation de la constitution, l'importance d'évoquerune loi non de moindre importance en l'occurrence, la loi organique 111.14 sur la région.

\section{2 la loi organique 111.14 sur la région}

\subsubsection{Le contexte}

Dans un souci de concrétisation de la haute volonté du chef suprême de l'Etat, le roi Mohammed $\mathrm{VI}^{8}$,et afinde réformerles structures étatiques sur la base d'une vision ambitieuse, un développement intégré et durable sur tous les plans structurants dignes d'un Etat moderneaspirant à un développement intégrée et durable, mettant en avant l'économique, le culturel, le social et l'environnemental,les autorités publiques compétentes avec le concours des académiciens et chercheurs,ont œuvré pour doter le Maroc d'une régionalisation avancée, d'essence principalement démocratique, érigeant la région au rang d'associé de l'Etat,la libérant ainsi de la tutelle excessive qui cabrait la propulsion de la région en tant qu'entité infra-étatique, autonomeet vouée de larges prérogatives, sans se départir du processus de décentralisation et de déconcentration conséquentes et effectiveset de bonne gouvernance.Il relevait donc de l'évidence dela consacrer dans la constitution de $2011^{9} \mathrm{et}$ de promulguer la loi relative à la région lui garantissant de nouveauxrôles et plus amples compétences.

\subsubsection{La consécration législative}

Tout en restant fidèle à la constitution, la loi organique 111-14 a mis en avant une multitude d'article réglementant les mécanismes participatifs, on cite d'emblée, l'article premier, qui stipule que «la présente loi organique fixe conformément à l'article 146 de la constitution: les conditionsde gestion démocratique par la région de ses affairesainsi que les conditions de l'exercice des citoyennes et citoyens et les associations du droit de pétition. ", le chapitre IV intitulé - des mécanismes participatifs de dialogue et de concertation, par conséquent, indiquerespectivement dansles articles 116 et 117que: «les conseils des régions mettent en place des mécanismes participatifs de dialogue et de concertation pour favoriser l'implication des citoyennes et citoyens, et des associations dans l'élaboration et le suivi des programmes de développement régional, selon les modalités fixés dans le règlement intérieur de la région. », «sont créées auprès de la région trois instances consultatives :la première en partenariat avec les acteurs de la société civile, chargé de l'étude des affaires régionales pour mettre en ouvre les principes de l'équité, de l'égalité des chanceset de l'approche genre. La deuxième pour étudier les questions relatives aux centres d'intérêt des jeunes, la dernière en partenariat avec les acteurs économiques de la région pour étudier les questions à caractère économique. », ensuite, le chapitre V intitulé: -Des conditions d'exercice par les citoyennes et les citoyens et les associations du droit de pétitionexplique les conditions et les modalités de l'exercice de ce droit dans ses articles 118,119,120,121et 122 que nous nous abstenons de citer vue leur langueur ${ }^{10}$.( se référer au texte intégral). On ne manquera pas de mentionner les deux autres lois organiques ${ }^{11}$ relatives aux deux autres échelons territoriaux, à savoir la province et la commune qui prévoient presque les mêmes mécanismes de l'approche participative dans le cadre du principe de subsidiarité.

A tort, ce serait de prétendre que le droit à la participation citoyennen'a pas jouit d'une consécration législative significative, mais ceci ne signifie pas non plus que la mise en pratique n'est pas susceptible d'imperfection voire de limites.

\section{LES CONTRAINTES DE LA MISE EN GEUVREDE L'APPROCHE PARTICIPATIVE}

Il nous incombe de noter que la participation citoyenne se conçoit partant de deux visions ou deux angles, suivant sans l'ombre d'un doute, des finalités disparates et tributaires de deux acteurs (l'Etat et la population), pour le premier, c'est un outil d'exercice de la démocratie, pour le second, elle est un mécanisme de reconnaissance de ce dernier comme étant partie intégrante de l'équation concourant à la décision politique. C'est la différence entre la simple formalité pour orner la facette politique et la ferme volonté d'impliquer les tiers de l'Etat dans la vie publique et la prise de décision.

L'approche participative est censée assurer par nature, l'équité, l'intégrité, la transparence et l'honnêteté des politiques qui en résultent; se sentant acteur dans le processus décisionnel, le simple citoyen ressent le sentiment d'appartenance à la communauté, et reprend confiance en son gouvernement et au système étatique dans son ensemble. Il n'en va pas de même en pratique, parce que L'approche participative est le plus souvent mal utilisée , mal justifiée ou inutile car la décision est déjà prise, ce qui engendre une crise de confiance de la part du citoyen envers son gouvernement et par conséquent des institutions, en même temps, le pouvoir 
lui-même juge les différentes procédures de participation comme une déperdition du temps, partant de la logique que les citoyens ne sont pas aptes à discuter de certains sujets avec des spécialistes, chose qui a été constatée dans les expériencesauxquelles on a pu assister sur invitationà l'échelon territorialle plus mineur( instance de l'équité, de l'égalité des chances et de l'approche participative).

Il va sans dire que la constitution et la loi organique qui s'en suit se doivent de permettre une réelle garantie au citoyen de jouir des droits qui y sont adoptés, seulement les articles de la constitution notamment le premier et le deuxième paragraphe de l'article 139 prévoyant des mécanismes participatifs de dialogue et de concertation et conférant le droit de pétition aux citoyennes et citoyens ont un aspect général et les modalités en sont assez ambigües, il relevait donc, de la loi organique 14-111 relative à la région àen expliciter et en préciser la portée à travers les chapitres 4 et 5 respectivement: les mécanismes participatifs du dialogue et de consultation et les conditions de dépôts des pétitions par les citoyennes et les citoyens et les associations, lesquels mécanismes semblent encore une fois alourdis par des procédures et des conditions peu encourageantes (nombre des signatures, désignation du représentant,le profil requis, modalités de mise en œuvre, ce qui soulève une vraie problématique sur la disposition du citoyen à s'aventurer dans ce terrain épineux et décourageant pour parvenir en finà faire entendre sa voix, on lui demande d'agir par procuration, autrement d'être à nouveau représenté . De surcroît, la loi est restée muette sur les mécanismes de vote de ces apports ou motions hétérogènes aux délibérations dans les sessions, parailleurs, la loi en question demeure peu convaincante quant aux différentes mesures et procédés qui peuvent en résulter et garantir au maximum la participation des citoyens à la prise de décision dans le cadre d'une vraie concertation publique au sein de vraies institutions et espaces publiques qui peuvent être érigés à cet effet. D'un autre côté, le concours des citoyens dans ce processus décisionnel est basé sur une prérogative consultative seulement, donc dépourvue de toute autorité ou contrainte.En fin, au Maroc, la loi et par récurrence les droitssont toujours mal diffusés, ce qui met une grande majorité des citoyensdans l'ignorance totale des droits qui leur sont impartis.

L'idée de faire participer divers acteurs sociaux à l'élaboration des politiques publiques n'est pas nouvelle en soi, mais si, dans d'autres pays elle est passé d'un processus élitiste à un espace de représentation important, au Maroc elle se base toujours sur invitation et non une représentation publique ouverte et inclusive essayant de cibler les différents groupes sociaux.La constitution de 2011et la loi organique conséquente sur la région, se veutelle vraiment impartiale et populaire ou non ?Existerait-il un exécutif au sein de la région capable de matérialiser démocratiquement l'esprit de cette loi organique ? Ou souffrirait-on encore une fois d'un déficit décisionnel privilégiant l'orgueil de l'élite élue au réel dialogue ? Le pouvoir en place est-il vraiment prédisposé à adopter une approche participative digne de ce nom ou se heurteronsnous encore une fois à un doute de sa part sur la pertinence d'associer les différents groupes à la prise de décisions, en attribuant l'échec des participations à la présence d'une trop grande diversité d'intérêts ?

Ajoutons à cela un élément psychique auquel se heurte la mise en pratique des mécanismes participatifs, il est ressenti à la fois du côté des autorités compétentes qui ont du mal accepter le concours de gens hétéroclites au système, et du côté du citoyen lui-même qui reste réticent, écœuréet sceptique par rapport à cette participation pour la simple raison qu'au final, c'est rarement qu'on lui donne raison car la puissance publique a prouvé plus d'une fois qu'elle s'accapare de la décision, le simple citoyen y est collé d'une manière illusoire appâté par ce miroir aux alouettes.

Tout compte fait,notre suspicion est accentuée tant par la paresse du pouvoir législatif que celle de la puissance publique, la censure et le harcèlement continu des citoyens à chaque manifestation publique prétendant à exprimer une opinion quelconque, laisse à désirer et élargit le fossé entre le citoyen et le pouvoir.

\section{CONCLUSION}

Sommes toutes, force est de soulever au terme de cette analyse qu'un Etat qui se veut démocratique et organisé, est appelé, plus que jamais, à créer un climat idoine à l'interdépendance réciproque avec le citoyen et matérialiser l'égalité de participation citoyenne comme unique issue pouvant assurer une influence des grandes orientations dans la conduite du pays, la simple déclaration d'œuvrer pour une bonne gouvernance n'est guère suffisante à garantir la démocratie dans ces aspects les plus concrets, mais encore faut-il que celle-ci soit le fruit d'une vraie volonté de faire participer le citoyen au processus décisionnel, les écarts et les disparités sociales ne cessent de s'accroître, on est toujours loin de la justice territoriale, peut-être il est temps de revoir et de reconsidérer les mécanismes de mise en œuvre de la participation citoyenne. 


\section{REFERENCES}

[1] Ph. Ardent Institutions Politiques et Droit Constitutionnel, $10^{\mathrm{e}}$ ed., 1998. Paris : Librairie générale de droit et de jurisprudence, E.J.A. p.176

[2] Loïc. Blondiaux: professeur des universités à l'I.E.P. de Lille, C.E.R.A.P.S., Lille-I, www.universalis.fr

[3] Op.cit., p., 178

[4] P. André, professeur à l'université de Montréal pierre Avec la collaboration de P. Martin et G. Lanmafankpotin

[5] H.Ghiat: 2016,(Chef Division des Affaires Juridiques et Relations Publiques,Ministère chargé des relations avec le gouvernement et la société civile marocain)

[6] Réglementé par la loi organique $n^{\circ}$ 14-64 fixant les conditions de présenter des motions en matière législative, promulgué par le dahir du 28 juillet 2016

[7] Réglementé par la loi organique $n^{\circ}$ 14-44 fixant les conditions de présenter des motions en matièrelégislative, promulgué par le dahir du 28 juillet 2016

[8] Discours Royal du 3 janvier 2010

[9] Constitution marocaine de 2011, l'article 1 et le titre IX

[10] La loi organique 111-14 sur les regions au Maroc

[11] Les lois organiques 112-14 et 113-14 sur les provinces et les communes au Maroc 